could be a novel therapeutic approach to reducing CHD risk in the general population.

\section{REFERENCES AND NOTES}

1. D. J. Rader, A. R. Tall, Nat. Med. 18, 1344-1346 (2012).

2. C. L. Haase et al., J. Clin. Endocrinol. Metab. 97, E248-E256 (2012)

3. R. Frikke-Schmidt et al., JAMA 299, 2524-2532 (2008).

4. B. F. Voight et al., Lancet 380, 572-580 (2012).

5. S. Acton et al., Science 271, 518-520 (1996).

6. M. Hoekstra, T. J. Van Berkel, M. Van Eck, World J. Gastroenterol. 16, 5916-5924 (2010).

7. N. Wang, T. Arai, Y. Ji, F. Rinninger, A. R. Tall, J. Biol. Chem. 273, 32920-32926 (1998).

8. Y. Ueda et al., J. Biol. Chem. 274, 7165-7171 (1999).

9. K. F. Kozarsky et al., Nature 387, 414-417 (1997).

10. Y. Ji et al., J. Biol. Chem. 274, 33398-33402 (1999)

11. M. L. Varban et al., Proc. Natl. Acad. Sci. U.S.A. 95, 4619-4624 (1998).

12. A. Rigotti et al., Proc. Natl. Acad. Sci. U.S.A. 94, 12610-12615 (1997).

13. M. Brundert et al., Arterioscler. Thromb. Vasc. Biol. 25, 143-148 (2005).

14. Y. Ueda et al., J. Biol. Chem. 275, 20368-20373 (2000).

15. K. F. Kozarsky, M. H. Donahee, J. M. Glick, M. Krieger, D. J. Rader, Arterioscler. Thromb. Vasc. Biol. 20, 721-727 (2000).

16. T. Arai, N. Wang, M. Bezouevski, C. Welch, A. R. Tall, J. Biol. Chem. 274, 2366-2371 (1999).

17. M. Van Eck et al., J. Biol. Chem. 278, 23699-23705 (2003).

18. B. Trigatti et al., Proc. Natl. Acad. Sci. U.S.A. 96, 9322-9327 (1999).

19. D. Huszar et al., Arterioscler. Thromb. Vasc. Biol. 20 1068-1073 (2000).

20. A. Braun et al., Circ. Res. 90, 270-276 (2002).

21. Y. Zhang et al., J. Clin. Invest. 115, 2870-2874 (2005).

22. T. M. Teslovich et al., Nature 466, 707-713 (2010).

23. A. Ghodsizadeh et al., Stem Cell Rev. 6, 622-632 (2010)

24. M. A. Cayo et al., Hepatology 56, 2163-2171 (2012).

25. S. K. Mallanna, S. A. Duncan, Curr. Protocol Stem Cell Biol. 26 (Unit 1G), 4 (2013).

26. K. Si-Tayeb et al., Hepatology 51, 297-305 (2010).

27. J. Babitt et al., J. Biol. Chem. 272, 13242-13249 (1997).

28. M. Viñals, S. Xu, E. Vasile, M. Krieger, J. Biol. Chem. 278 5325-5332 (2003).

29. D. Calvo, D. Gómez-Coronado, M. A. Lasunción, M. A. Vega, Arterioscler. Thromb. Vasc. Biol. 17, 2341-2349 (1997)

30. C. C. Schwartz, J. M. VandenBroek, P. S. Cooper, J. Lipid Res. 45. 1594-1607 (2004)

31. M. Vergeer et al., N. Engl. J. Med. 364, 136-145 (2011)

32. R. B. Hildebrand et al., Arterioscler. Thromb. Vasc. Biol. 30 1439-1445 (2010)

33. L. Lagrost et al., Arterioscler. Thromb. 13, 815-825 (1993).

34. H. E. Miettinen, H. Rayburn, M. Krieger, J. Clin. Invest. 108, 1717-1722 (2001)

35. M. Hoekstra et al., J. Lipid Res. 49, 738-745 (2008).

36. M. Hoekstra et al., J. Lipid Res. 50, 1039-1046 (2009)

37. O. Kocher et al., J. Biol. Chem. 278, 52820-52825 (2003).

38. C. Mineo, P. W. Shaul, Curr. Opin. Lipidol. 23, 487-493 (2012).

39. A. Rigotti, H. E. Miettinen, M. Krieger, Endocr. Rev. 24, 357-387 (2003).

\section{ACKNOWLEDGMENTS}

We appreciate the participation and support of participants of the deep clinical phenotyping studies. We thank E. Mohler for assistance in

interpretation of cIMT results and J. Billheimer and E. Pashos for helpful discussions. We also acknowledge J. Tabita-Martinez for expert assistance with clinical phenotyping studies. This work was supported in part by an award from the National Center for Research Resources (grant TL1RR024133) and National Center for Advancing Translational Sciences of the NIH (grant TL1R000138) to support patient recruitment. D.B.L. was supported by a fellowship from the Doris Duke Charitable Foundation. S.K. has financial relationships with Novartis, Aegerion, Bristol-Myers Squibb, Sanofi, AstraZeneca, Alnylam, Eli Lilly, Leerink Partners, Merck, Catabasis, Regeneron Genetic Center, San Therapeutics, and Celera. H.S. has financial relationships with MSD Sharp and Dohme, Sanofi-Aventis, and Amgen. S.B. has financial relationships with Boehringer Ingelheim, Bayer, Novartis, Roche, and Thermo Fisher. N.S has financial relationships with Amgen, Sanofi, Astrazeneca, and MSD Sharp and Dohme. A.K. has a financial relationship with Amgen. J.D. has a financial relationship with Novartis. A.T.-H. has financial relationships with Eli Lilly and LGC Genomics. Sequencing data have been deposited in GenBank (SRX1458096).
Genotyping data have been deposited in the Gene Expression Omnibus (GSE76065).

SUPPLEMENTARY MATERIALS

www.sciencemag.org/content/351/6278/1166/suppl/DC1

Materials and Methods

Supplementary Text
Figs. S1 to S7

Table S1

References (40-76)

Consortia and Study Author Lists

1 September 2015; accepted 7 January 2016

10.1126/science.aad3517

\title{
Wavelike charge density fluctuations and van der Waals interactions at the nanoscale
}

\author{
Alberto Ambrosetti, ${ }^{1,2}$ Nicola Ferri, ${ }^{1}$ Robert A. DiStasio Jr., ${ }^{3 *}$ Alexandre Tkatchenko ${ }^{1,4 *}$
}

Recent experiments on noncovalent interactions at the nanoscale have challenged the basic assumptions of commonly used particle- or fragment-based models for describing van der Waals (vdW) or dispersion forces. We demonstrate that a qualitatively correct description of the vdW interactions between polarizable nanostructures over a wide range of finite distances can only be attained by accounting for the wavelike nature of charge density fluctuations. By considering a diverse set of materials and biological systems with markedly different dimensionalities, topologies, and polarizabilities, we find a visible enhancement in the nonlocality of the charge density response in the range of 10 to 20 nanometers. These collective wavelike fluctuations are responsible for the emergence of nontrivial modifications of the power laws that govern noncovalent interactions at the nanoscale.

\section{I} he assembly of complex nanostructures and biological systems from simpler building blocks is often driven by noncovalent van der Waals (vdW) or dispersion interactions that arise from electrodynamic correlations between instantaneous charge fluctuations in matter $(1,2)$. The influence of vdW forces extends well beyond binding energies and encompasses the structural $(3,4)$, mechanical $(5,6)$, spectroscopic (7), and even electronic (8) signatures of condensed matter. A common way to characterize vdW interactions is by power laws in the distance $D$ between two or more objects (e.g., atoms, molecules, nanostructures, surfaces, or solids); the most familiar is arguably the LennardJones potential, which is characterized by a shortrange repulsive wall with a $D^{-12}$ dependence and a long-range attractive tail with a $D^{-6}$ dependence. Even a slight variation in these power laws can have a profound impact on observed properties and therefore demands an accurate, physically sound theoretical description.

Thus far, both our conceptual understanding of vdW interactions and the quantitative models widely used for describing these quantum mechanical phenomena are primarily rooted in low-order intermolecular perturbation theory (IPT), wherein

${ }^{1}$ Fritz-Haber-Institut der Max-Planck-Gesellschaft, D-14195 Berlin, Germany. ${ }^{2}$ Dipartimento di Fisica e Astronomia, Università degli Studi di Padova, 35131 Padova, Italy. ${ }^{3}$ Department of Chemistry and Chemical Biology, Cornell University, Ithaca, NY 14853, USA. ${ }^{4}$ Physics and Materials Science Research Unit, University of Luxembourg, L-1511 Luxembourg.

${ }^{*}$ Corresponding author. E-mail: distasio@cornell.edu (R.A.D.); alexandre.tkatchenko@uni.lu (A.T.)
vdW binding originates from the interactions between transient local multipoles (9), and macroscopic Lifshitz theory (10). Although IPT-based approaches have had enormous success in describing vdW binding in (small) gas-phase molecular systems $(11,12)$, recent advanced experimental techniques have produced several findings that are challenging the basic assumptions of IPT and macroscopic approaches for nanostructured materials, and are strongly indicative that even our qualitative understanding of these interactions is incomplete and needs to be substantially revised (13). Examples of such experimental observations include (i) ultra-long-range vdW interactions extending up to tens of nanometers into heterogeneous dielectric interfaces $(14,15)$, (ii) complete screening of the vdW interaction between an atomic force microscope (AFM) tip and a $\mathrm{SiO}_{2}$ surface by the presence of one or more layers of graphene adsorbed on the surface (16), (iii) superlinear sticking power laws for the self-assembly of metallic clusters on carbon nanotubes with increasing surface area (17), and (iv) nonlinear increases in the vdW attraction between homologous molecules and an $\mathrm{Au}$ (111) surface as a function of molecular size (18). Satisfactory theoretical explanations for these experimental findings either require ad hoc modifications to IPT [(iii) and (iv)] or are inherently outside the domain of applicability of IPT [(i) and (ii)].

To address these issues, we note that the spatial extent of the instantaneous charge density fluctuations responsible for vdW interactions depends rather sensitively on the nature and character of the occupied-to-virtual transitions of the 
valence electrons in a given molecule or material. In this regard, the inherent delocalization characteristic of low-dimensional metallic systems leads to a remarkably slow decay in the interaction energies between metallic chains and layers as a function of their separation (19-21), which represents a substantial modification of the "conventional" asymptotic behavior of the vdW interaction energy that dominates the binding at very large distances (e.g., beyond 10 to $20 \mathrm{~nm}$ in bilayer graphene) (22). Moreover, Misquitta et al. $(23,24)$ demonstrated that upon closure of the band gap, semiconducting nanowires may also exhibit unconventional power laws as a function of the interwire distance $D$, namely $\sim D^{-2}$ at intermediate separations, followed by asymptotic convergence to the pairwise-additive $D^{-5}$ limit for large interwire separations.

Here, we revise and extend these seminal asymptotic results to the finite distance regime between nanostructures, because interactions at such distances (as opposed to asymptotic separations) determine the structural, mechanical, spectroscopic, and (opto)electronic properties at the nanoscale. By considering a series of relevant zero-, one-, and two-dimensional systems that include proteins, carbyne-like wires, nanotubes, graphenic layers, and $\mathrm{MoS}_{2}$, we show that a qualitatively correct description of vdW interactions between nonmetallic nanostructures can only be attained by accounting for the wavelike nature of charge density fluctuations in such systems. In doing so, our analysis extends well beyond the low-order particle- or fragment-based IPT paradigm and provides further insight into the roles played by dimensionality, topology, and polarizability in determining the magnitude of these fundamental forces at the nanoscale.

\section{The many-body dispersion approach}

In systems where electrons are well described by a localized representation of the occupied space (e.g., systems with finite band gaps), collective charge density fluctuations stem from the dynamically correlated motions of local dipolar excitations. Accordingly, we project the valence electronic response in a given nucleo-electronic system onto a set of $N$ interacting atomic response functions, as outlined by the many-body dispersion (MBD) approach (25). This scheme has been applied with great success to the computation of polarizabilities (26) and dispersion interactions in weakly bound systems $(27,28)$, and has recently been extended to arbitrary nonmetallic molecules, solids, and nanostructures through the use of spatially distributed polarizabilities $(25,29)$. The advantage of the MBD method resides in an efficient and accurate quantum mechanical parameterization of the valence electronic response in terms of coupled atomic dipolar fluctuations $(30,31)$, allowing for a chemically accurate treatment of molecules and extended systems when used in conjunction with state-of-the-art exchange-correlation (XC) functionals in density functional theory $(3,4,7,29,32-35)$.

Within the MBD framework, the long-range correlation energy $E_{\mathrm{vdW}}^{\mathrm{LR}}$ is computed via the ad- iabatic connection fluctuation-dissipation theo$\operatorname{rem}(30,36)$ as

$$
E_{\mathrm{vdW}}^{\mathrm{LR}}=-\frac{1}{2 \pi} \int_{0}^{\infty} d \omega \int_{0}^{1} d \lambda\left[\operatorname{Tr}\left(\chi_{\lambda}-\chi_{0}\right) v\right]
$$

in which $\chi_{0}$ is the bare response function for a system of noninteracting atoms and $\chi_{\lambda}$ is the interacting response function "dressed" by the rescaled and range-separated $(25,29)$ Coulomb interaction, $\lambda v$. [We adopt the atomic units convention of $m_{\mathrm{e}}=\hbar=e=1 /\left(4 \pi \varepsilon_{0}\right)=1$, where $m_{\mathrm{e}}$ is the mass of the electron, $\hbar$ is the Planck constant divided by $2 \pi, e$ is the elementary charge, and $\varepsilon_{0}$ is the permittivity of free space.] Within the dipole approximation, the energy expression in Eq. 1 can be computed exactly and is equivalent to diagonalization of the coupled dipolar Hamiltonian (30),

$$
\begin{aligned}
\hat{H}_{\mathrm{MBD}}= & -\frac{1}{2} \sum_{p=1}^{N} \nabla_{\mathbf{d}_{p}}^{2}+\frac{1}{2} \sum_{p=1}^{N} \omega_{p}^{2} \mathbf{d}_{p}^{2}+ \\
& \sum_{p>q}^{N} \omega_{p} \omega_{q} \sqrt{\alpha_{p}^{0} \alpha_{q}^{0}} \mathbf{d}_{p} \mathbf{T}_{p q} \mathbf{d}_{q}
\end{aligned}
$$

in which each atom $p$ is characterized by a static dipole polarizability $\alpha_{p}^{0}$ and a characteristic excitation frequency $\omega_{p}$, and $\mathbf{d}_{p}$ represents the mass-weighted displacement of atom $p$ from its equilibrium position $\mathbf{R}_{p}$. The first two terms in this Hamiltonian correspond to the single-particle kinetic and potential energies, respectively; the last term describes the coupling between atoms $p$ and $q$ via the dipole-dipole interaction tensor $\mathbf{T}_{p q}=\nabla_{\mathbf{R}_{p}} \otimes \nabla_{\mathbf{R}_{q}} v\left(R_{p q}\right)$, where $v\left(R_{p q}\right)$ is the Coulomb interaction at the interatomic distance $R_{p q}=\left|\mathbf{R}_{p}-\mathbf{R}_{q}\right|(30) . E_{\mathrm{vdW}}^{\mathrm{LR}}$ is then computed as the energetic difference between the eigenfrequencies of the collective modes of the fully interacting system, $\bar{\omega}_{i}$, and the characteristic excitation frequencies of the isolated atoms, $\omega_{p}$.

\section{Dipole waves in one-dimensional carbyne wires}

We begin our investigation into the nature of vdW interactions at the nanoscale with a detailed analysis of the collective charge density fluctuations sustained in a linear one-dimensional (1D) carbyne-like atomic wire comprising 2000 atoms (and subject to periodic boundary conditions). From Fig. 1A, we first note that such finite atomic wires are characterized by a set of very-low-energy collective eigenmodes (albeit with nonzero energy eigenvalues). In this regard, the minimum energy (gap) observed in these modes approaches the independent atom value of $\omega_{\mathrm{C}}=0.43 \mathrm{Ha}$ (Hartree units) at large C-C distances $d_{\mathrm{C}-\mathrm{C}}$, but decreases to approximately $1 / 20$ th of this value at $d_{\mathrm{C}-\mathrm{C}}=1.2 \AA$, the C-C bond length in acetylene. Because of charge conservation, the quantity $\bar{\alpha}_{i}^{0} \bar{\omega}_{i}^{2}$ (where $\bar{\alpha}_{i}^{0}$ and $\bar{\omega}_{i}^{2}$ denote the static dipole polarizability and resonant frequency of the $i$ th eigenmode, respectively) must be equivalent for every MBD eigenmode, as initially set by the independent atomic value of $\alpha_{\mathrm{C}}^{0} \omega_{\mathrm{C}}^{2}$. Hence, the lowest-energy eigenmode can be characterized by a substantially increased polarizability (i.e., up to $\sim 400$ times that of a single $\mathrm{C}$ atom), which is strongly indicative of a marked delocalization of the charge density fluctuations over the entire wire. In this case, the low dimensionality and particular topology of this nanostructure is crucial for sustaining coherent delocalized fluctuations along the length of the wire. As depicted in Fig. 1, B and C, these fluctuations result from strongly enhanced (head-to-tail) dipole-dipole coupling along the longitudinal axis of the atomic wire, which leads to substantial anisotropy in the associated polarizability tensor (37).

To further expand on these observations, we analyzed the low-energy spectrum of the collective MBD eigenmodes in this low-dimensional nanostructure. As illustrated in Fig. 1, B and C, these modes correspond to coherent dipolar fluctuations aligned along the entire wire with negligible components orthogonal to the longitudinal axis. Consistent with a normal mode analysis of a dipolar-coupled system with this underlying topology, the higher-energy modes correspond to polarization waves with an increasing number of nodes. At the highest energies (i.e., beyond the shoulder in the spectrum of Fig. 1A), these eigenmodes have sizable transverse components and therefore make a negligible contribution to the coherent dipolar fluctuations along the wire.

Upon compression of the carbyne wire (i.e., by varying $d_{\mathrm{C}-\mathrm{C}}$ from 3.0 to $1.2 \AA$ ), the gap in the eigenvalue spectrum becomes visibly reduced and is accompanied by a corresponding increase in the slope of the dispersion curves (Fig. 1A). A gapless metallic dispersion (21) is thus approached, but never reached, in the MBD model-a fact that we attribute to the intrinsic charge confinement of the valence atomic responses. A flat dispersion (approached only in the limit $d_{\mathrm{C}-\mathrm{C}} \rightarrow \infty$ ) would indicate a localization of the system response; with every eigenmode degenerate in energy, single-atom dipolar fluctuations would occur along the wire and result in a decorrelation of the atomic susceptibilities. Conversely, we observe from Fig. 1A that intrawire interactions can also induce nontrivial dispersion in the eigenvalue spectrum despite the absence of explicitly delocalized electrons, implying a marked nonlocality in the collective dipolar response within the MBD model. This collective behavior stems from a subtle interplay between the kinetic and potential energy operators in the MBD Hamiltonian (see. Eq. 2). Upon compression of the carbyne wire, the kinetic energy term increases relative to the potential energy terms, leading to large-amplitude oscillatory motions induced by the presence of many-body kinetic energy contributions. In addition, an analysis of the exact two-atom MBD solution reveals an underlying dependence of $E_{\mathrm{vdW}}^{\mathrm{LR}}$ on the quantity $\alpha_{\mathrm{C}} d_{\mathrm{C}-\mathrm{C}}^{-3}$; as such, the effect of varying the interatomic distance is expected to be qualitatively equivalent to an inversely proportional modification of the polarizability.

\section{vdW interactions between carbyne wires}

The analysis presented above already provides strong evidence of the importance of wavelike charge density fluctuations in accounting for vdW interactions in low-dimensional nonmetallic nanostructures. To investigate this aspect further, we 
A

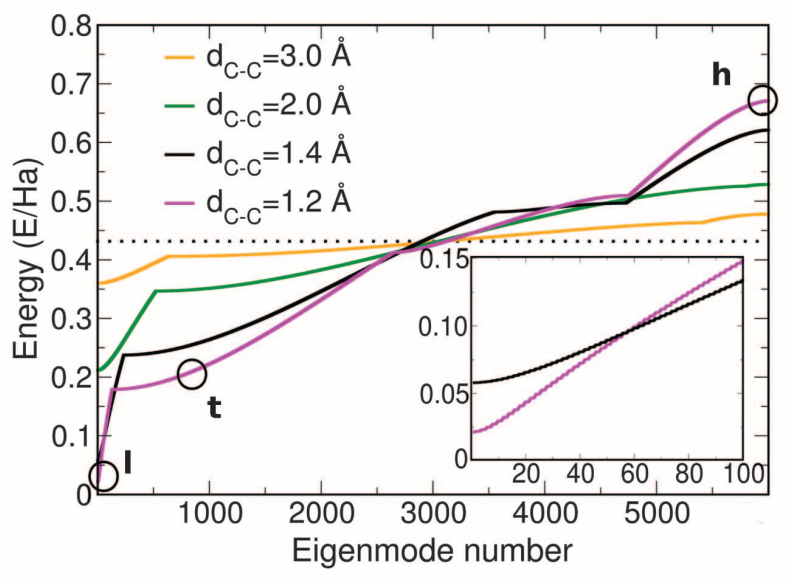

Fig. 1. Charge density fluctuations in carbyne wires. (A) Energy eigenvalue spectrum corresponding to the $3 \mathrm{~N}$ collective eigenmodes of the many-body dispersion (MBD) model $(25,29)$ plotted in ascending order for $1 \mathrm{D}$ carbynelike atomic wires comprising $N=2000 \mathrm{C}$ atoms (subject to periodic boundary conditions) and values of the interatomic $\mathrm{C}-\mathrm{C}$ distance $\mathrm{d}_{\mathrm{C}-\mathrm{C}}$ ranging from $1.2 \AA$ (the C-C bond length in acetylene) to $3.0 \AA$. For reference, the independent atom value of $\omega_{C}=0.43 \mathrm{Ha}$ is denoted by the dotted line. The inset shows a more detailed view of the low-energy portion of the spectrum, with the same units. (B) Schematic illustration of several representative low-energy (I), transverse (t), and high-energy (h) collective MBD eigen-
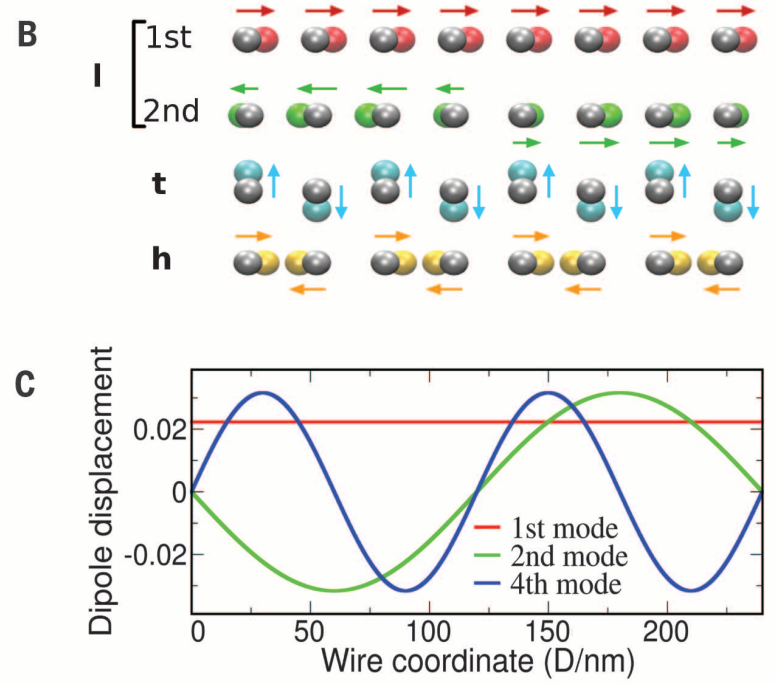

modes, as indicated in (A). The colored spheres represent dipole displacements with respect to the equilibrium atomic positions (depicted in gray). (C) Longitudinal dipole displacements of several low-energy collective MBD eigenmodes (in arbitrary units) with respect to the wire coordinate for $d_{\mathrm{C}-\mathrm{C}}=$ 1.2 $\AA$. Because of the inversion symmetry of this system, every eigenmode is doubly degenerate except for the lowest-energy longitudinal mode (depicted in red). Consistent with a normal mode analysis of a dipolar-coupled system with this underlying topology, the number of nodes present in a given MBD eigenmode is directly correlated with its relative location in the energy eigenvalue spectrum.

Fig. 2. Power laws for interwire vdW interactions. Interwire interaction energy power-law exponents (46) for two parallel 1D carbyne-like atomic wires as a function of the interwire separation $D$ and several values of $d_{\text {C-C }}$ computed using the MBD model. Over a range of relevant nanoscale distances, there are strong deviations from the well-known asymptotic power law of $D^{-5}$, as predicted by widely used pairwise-

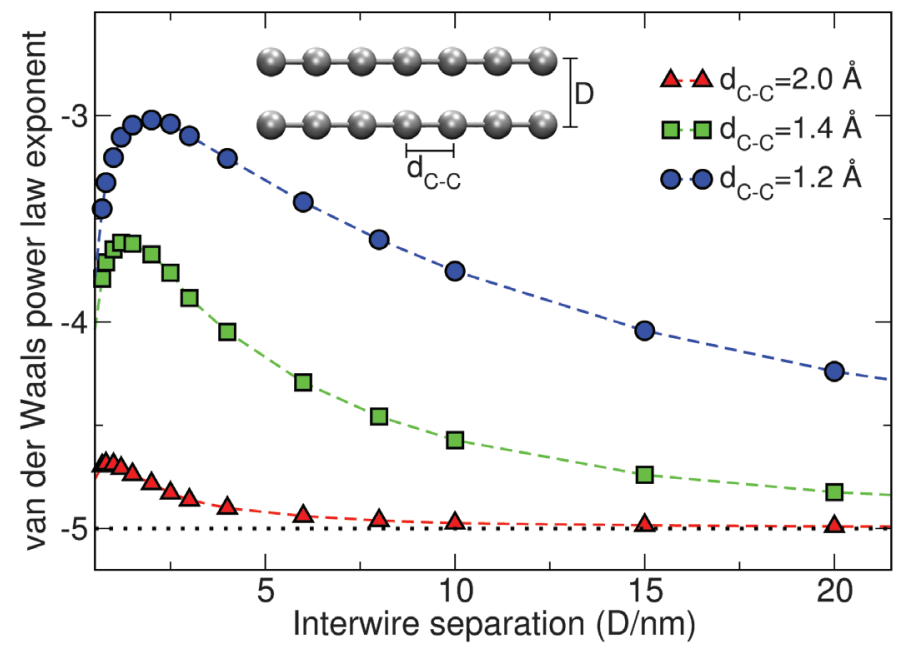

additive vdW models (denoted by the dotted line).

now consider the case of two mutually interacting parallel carbyne-like wires. In Fig. 2, the resulting vdW power-law exponents are plotted as a function of the interwire separation $D$ (for several values of $d_{\mathrm{C}-\mathrm{C}}$ ); they exhibit strong deviations from the asymptotic $D^{-5}$ behavior predicted by widely used vdW approaches based on pairwise additivity. This macroscopic effect goes beyond a simple renormalization of Hamaker constants and results from a theoretical account of both intra- and interwire many-body vdW interactions on an equal footing. As seen in Fig. 2, these modifications to the vdW interaction energy power laws become even more pronounced upon compression of the carbyne wire, reaching $\sim D^{-3}$ for interwire separations between 1 and $3 \mathrm{~nm}$ (with $d_{\mathrm{C}-\mathrm{C}}=1.2 \AA$ ), but still not converging to the pairwise-additive asymptote at interwire separations exceeding $20 \mathrm{~nm}$.

Such power-law modifications are particularly relevant at the nanoscale and can be understood as resulting from long-range electrodynamic coupling between the collective longitudinal modes (dipole waves) delocalized along the entirety of each carbyne-like wire. These wavelike charge density fluctuations are simply absent in localized particle- or fragment-based models for vdW interactions, despite being crucial for an accurate theoretical description of these fundamental interactions in low-dimensional nonmetallic nanostructures. These findings resemble the results obtained with approximate many-body models of metallic chains that explicitly account for the delocalized wavelike nature of metallic electrons $(5,21,23,38)$, which implies that a collective dipolar response with markedly nonlocal character can also be used to predict the emergence of wavelike dynamical electron correlation in nonmetallic systems.

To better understand the influence of these fluctuations on the vdW interaction between carbyne wires, we now consider an analytical model for this interaction in more detail. At sufficiently large $D$, the interwire Coulomb interaction in the continuum approximation is given by $w_{12}=$ $2 K_{0}(q D)$, where $K_{0}$ is a modified (or hyperbolic) Bessel function of the second kind and $q$ is the magnitude of the wave vector parallel to the longitudinal wire axis (21). Within the randomphase approximation, one can account for the effects of the interwire coupling on $\chi_{1}$, the response function of a single wire, via $\chi_{1}^{2} w_{12}^{2}=1$, which predicts a splitting of $\bar{\omega}(q)$ into eigenfrequencies that correspond to coupled dipolar fluctuations that are either aligned $\left(\bar{\omega}_{+}\right)$or antialigned $\left(\bar{\omega}_{-}\right)$with respect to the two parallel wires [i.e., $\bar{\omega}_{ \pm}(q)=\bar{\omega}(q) \sqrt{1 \pm \bar{\alpha}^{0}(q) \mathbf{T}(q)}$, wherein $\mathbf{T}(q)$ is the interwire dipole-dipole interaction derived from $w_{12}$ ]. Neglecting the higher-energy transverse eigenmodes that provide smaller contributions 
to the interwire interaction, the interaction energy between carbyne-like wires of length $L$ can then be computed in the nonretarded regime as

$$
E_{\mathrm{vdW}, 12}^{\mathrm{LR}}=\frac{L}{4 \pi} \int d q\left[\bar{\omega}_{+}(q)+\bar{\omega}_{-}(q)-2 \bar{\omega}(q)\right]
$$

which in turn can be expanded to second order in the interaction at large $D$, yielding

$$
E_{\mathrm{vdW}, 12}^{\mathrm{LR}} \approx-\frac{\left(\alpha_{\mathrm{C}}^{0} \omega_{\mathrm{C}}^{2}\right)^{2} L}{4 \pi d_{\mathrm{C}-\mathrm{C}}^{2}} \int d q \frac{K_{0}^{2}(q D) q^{4}}{\bar{\omega}^{3}(q)}
$$

A $q$-space decomposition of the integrand in Eq. 4 is provided in Fig. 3 and illustrates that the power law governing the interwire interaction energy has a nontrivial dependence on $D$ that originates from a summation over individual reciprocal-space contributions, each of which corresponds to a normal mode of the system with a different characteristic decay rate. This observation is in stark contrast to the determination of the interwire interaction energy from approximate particle- or fragment-based pairwise approaches, in which each contribution has the same $D^{-5}$ power-law decay. As such, this analysis is also strongly indicative that the complex power-law exponents displayed in Fig. 2 can only be correctly described by models that allow for delocalized wavelike fluctuations of the charge density. In this regard, even state-of-the-art nonlocal XC functionals $(39,40)$, which describe long-range electron correlation effects in a pairwise-additive fashion, would completely miss the interaction energy profiles in Fig. 2.

To gain a more detailed understanding of the power-law exponents displayed in Fig. 2, we note that the asymptotic behavior of the interwire interaction energy is largely influenced by $\bar{\omega}(q)$, the single-wire dispersion appearing in the integrand of Eq. 4. In fact, a first estimate of the decay rate of $E_{\mathrm{vdW}, 12}^{\mathrm{LR}}$ can be obtained by fitting the dispersion law with a single exponent-that is, as $\bar{\omega}(q) \propto q^{\delta}$-which directly leads to the finding that $E_{\mathrm{vdW}, 12}^{\mathrm{LR}} \propto D^{-5+3 \delta}$ upon variable substitution of $q^{\prime}=q / D$. Physically speaking, the quantity $K_{0}(q D)$ governs the interwire interaction by introducing a momentum cutoff at $q \approx 1 / D$, and the resultant integration over the infinitesimal contributions $q^{4} / \bar{\omega}^{3}(q)$ up to this momentum cutoff controls the scaling of $E_{\mathrm{vdW}, 12}^{\mathrm{LR}}$ with respect to $D$. In particular, a linear plasmon dispersion leads (up to logarithmic corrections) to a $D^{-2}$ decay (21) in metallic wires, whereas the slightly sublinear dispersion observed here for $d_{\mathrm{C}-\mathrm{C}}=1.2 \AA$ (see the inset in Fig. 1A after the initial energetic gap) is consistent with a $\sim D^{-2.8}$ power law and is in qualitative agreement with the numerical evidence provided by the MBD model in Fig. 2. Despite this initial deviation from $D^{-5}$, the power law at large distances again tends toward the pairwise-additive asymptote, although deviations still persist at interwire separations greater than $20 \mathrm{~nm}$. A direct account of this phenomenon follows from Fig. 3: Because of the decay of $K_{0}(q D)$ with respect to the quantity $q D$, only very small values of $q$ will contribute to the interwire interaction energy at large $D$ (see Eq. 4). In fact, only those wave vectors near the gap [in which $\omega(q) \approx$ constant] will be of relevance in recovering the asymptotic $D^{-5}$ limit. Hence, the pairwise-additive limit can only be approached in the presence of a flat dispersion, wherein the localization of the system response to single-atom dipolar fluctuations occurring along the individual wires would actually validate the fundamental particle- or fragment-based assumption that the vdW interaction energy can be described as a summation over induced atomic dipole contributions.

Note that the results of the above analysis are quite general and are not simply intrinsic properties of the MBD coupled dipolar Hamiltonian (Eq. 2). The same conclusions are observed when the qualitatively different tight binding (TB) model (23) is used for the single wire response. Within this approach, the individual atomic wires are described by a two-site nearest-neighbor interaction that can be tuned (by manipulating the $\beta_{1}$ and $\beta_{2}$ free-parameter space) to reproduce both the metallic (symmetric interactions via $\beta_{2} / \beta_{1}=1$ ) and insulating (asymmetric/single-sided interactions via $\beta_{2} / \beta_{1}=0$ ) limits. A second-order perturbative treatment of the interwire interaction energy leads

to power-law exponents that describe these two respective limits-that is, $\sim D^{-2}$ for metallic nanowires and $\sim D^{-5}$ for insulating nanowires with flat energy bands-as depicted in Fig. 4. By analogy to the MBD model, the TB response becomes markedly nonlocal in the metallic limit, the regime in which the largest deviations from the pairwise-additive $\sim D^{-5}$ power law are expected. Conversely, a substantial localization of the response is recovered in the insulating limit, in which the pairwise-additive approximation becomes asymptotically valid. Comparisons between the power-law exponents provided in Figs. 2 and 4 demonstrate semiquantitative agreement between the qualitatively different MBD and TB models, which is strongly indicative of the universality of the influence of wavelike charge density fluctuations on vdW interactions between nanostructures.

\section{vdW interactions between complex nanostructures}

Although the results above were obtained for model 1D carbyne-like wires, our calculations suggest that collective charge density fluctuations will also strongly influence the behavior of strained

Fig. 3. Analytical model for interwire vdW interactions. A reciprocal-space decomposition analysis of the integrand $K_{0}^{2}(q D) q^{4} / \bar{\omega}^{3}(q)$, which is required for computing the second-order interwire interaction energy in Eq. 4. Assuming linear dispersion, the decay rate of the integrand with respect to $D$ exhibits a nontrivial dependence on the momentum $q$ (in $\mathrm{nm}^{-1}$ ), which is responsible for the observed power laws. At large $D$, only small $q$ contributions substantially influence $E_{\mathrm{vdW}, 12}^{\mathrm{LR}}$, so that $D$ effectively determines the scale of relevant momenta. In this plot, the integrand has been rescaled by the constant $\bar{\omega}(0)^{3}$ and the corresponding color scale is provided in atomic units.

Fig. 4. The tightbinding model for interwire vdW interactions. Interwire interaction energy power-law exponents (46) are shown for two parallel $1 \mathrm{D}$ carbyne-like atomic wires as a function of the interwire separation $D$ computed using the tight-binding (TB) model $(23,46)$. With $d_{\mathrm{C}-\mathrm{C}}=$ $2.0 \AA$, the two limiting cases of insulating $\left(\beta_{2} / \beta_{1}=0\right)$ and metallic $\left(\beta_{2} / \beta_{1}=1\right)$ nanowires were considered, along with an intermediate

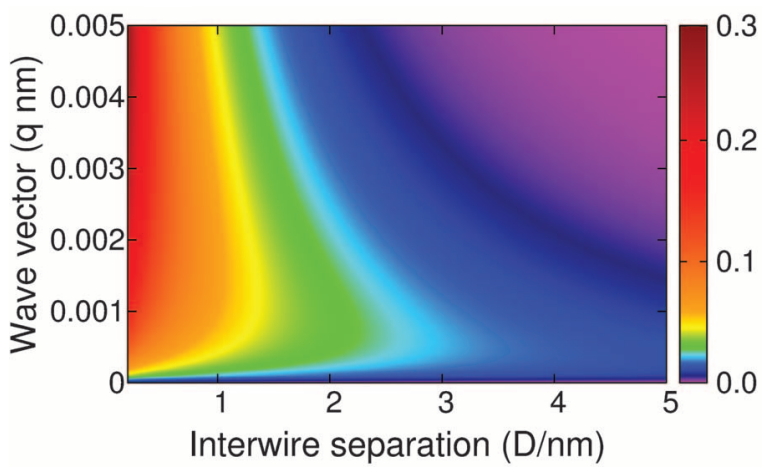

case $\left(\beta_{2} / \beta_{1}=0.96\right)$. As previously observed with the qualitatively different MBD model (see Fig. 2 ), there are strong deviations from the asymptotic $D^{-5}$ power law (denoted by the dotted line) over a wide range of relevant nanoscale distances.

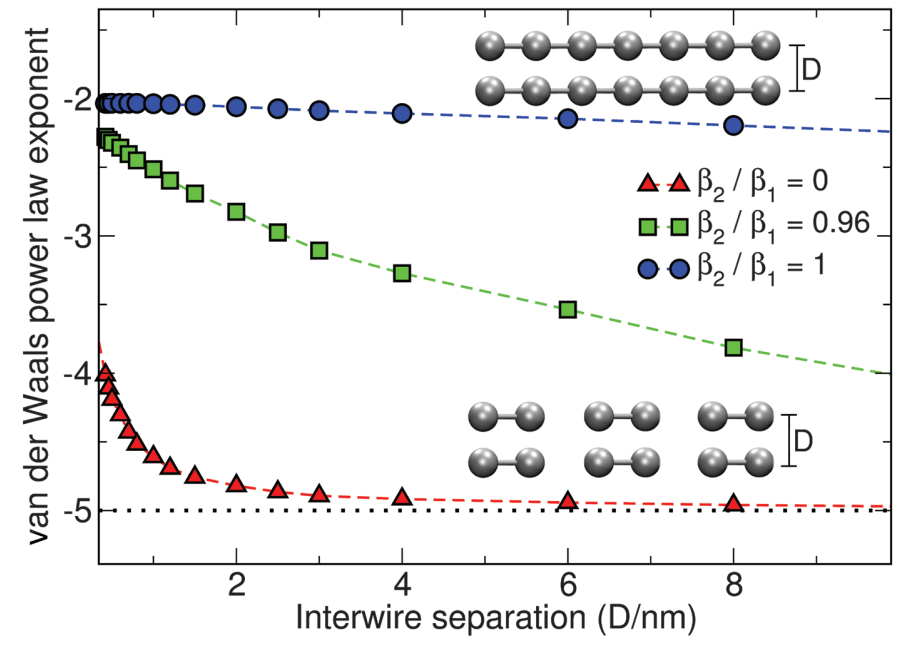


hydrocarbon polymers and inorganic wires made from polarizable elements such as phosphorus, silicon, or germanium. To further elucidate the roles of dimensionality, topology, and polarizability on determining vdW interaction power laws across distances relevant at the nanoscale, we carried out a study of layered two-dimensional (2D) materials, including graphenic sheets of varying polarizability as well as bilayer $\mathrm{MoS}_{2}$. Although a smaller reduction in the power-law exponent is expected in bilayer graphene because of its higher dimensionality, a $D^{-3.5}$ decay was found for interlayer distances of 0.6 to $3.0 \mathrm{~nm}$, with convergence to the conventional $D^{-4}$ power law only achieved for interlayer distances well beyond $10 \mathrm{~nm}$ (Fig. 5). In general, the interlayer interaction power-law exponents for bilayer graphene were intermediate between $D^{-3}$ and the pairwiseadditive $D^{-4}$ asymptote, which are induced by gapless $\pi$ states and are expected to be valid in the case of finite-gap doped graphene $(41,42)$. Increasing the polarization response of the graphenic sheets to effectively model highly polarizable $2 \mathrm{D}$ materials leads to substantial delocalization in the system response; for $\alpha_{\mathrm{C}}^{0}=50 \mathrm{bohr}^{3}$, the power law exhibited an extended plateau

Fig. 5. Power laws for interlayer vdW interactions. Interlayer interaction energy power-law exponents (46) for two parallel 2D graphenic layers and $\mathrm{MoS}_{2}$ as a function of the interlayer separation $D$ computed using the MBD model. To investigate the behavior of these power laws in highly polarizable 2D materials, we considered several values of $\alpha_{C}^{0}$ for the graphenic layers, rang-

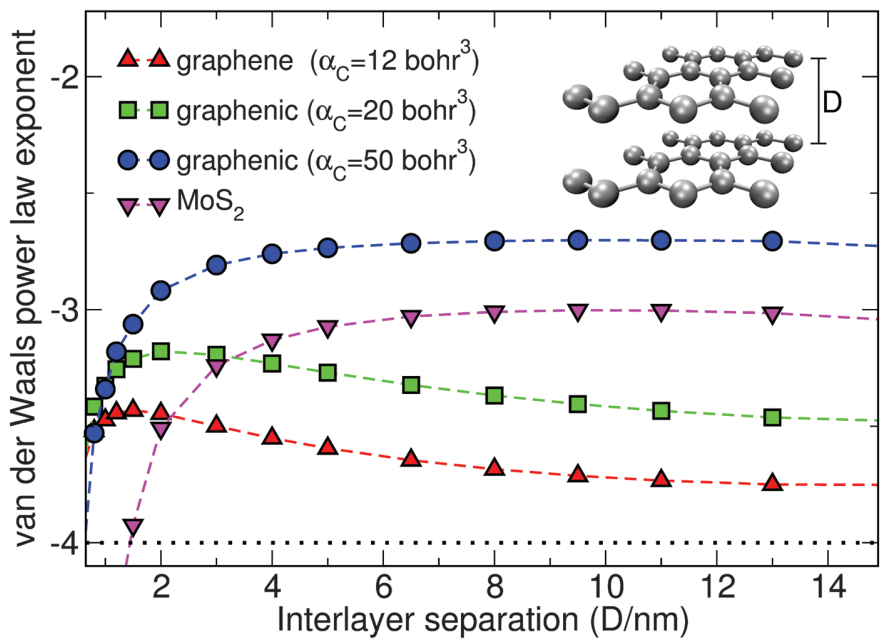

ing from $12 \mathrm{bohr}^{3}$ (the static atomic dipole polarizability of a C atom) to $50 \mathrm{bohr}^{3}$. As found above for the case of interacting parallel 1D nanowires over a range of relevant nanoscale distances, there are substantial deviations from the asymptotic $D^{-4}$ power law predicted by pairwise-additive vdW approaches (denoted by the dotted line).

Fig. 6. Power laws for vdW interactions between complex nanostructures. Interaction energy power-law exponents (46) computed using the MBD model for two parallel $(3,3)$ carbon nanotubes and a wire-protein nanostructure (depicted in insets) as a function of the respective centerof-mass separations $D$. As observed above for interacting $1 D$ nanowires and $2 \mathrm{D}$ layered materials, we report substantial deviations in the decay rate of the MBD interaction energy in such nanostructures when compared against the pairwise-additive asymptote of $D^{-5}$ (denoted by the dotted line). The ratio of the MBD and pairwise (12) interaction energies $\Delta E_{\mathrm{vdW}}^{\mathrm{LR}} / \Delta E_{\mathrm{vdW}}^{\mathrm{LR}(2)}$ are provided for select values of $D$; the growth of this ratio with $D$ is another indication of the nontrivial coupling between delocalized charge density fluctuations sustained across these nanostructures. between $D^{-2.5}$ and $D^{-3}$ at interlayer separations from $1.5 \mathrm{~nm}$ to well beyond $10 \mathrm{~nm}$-distances that are again quite relevant to interactions at the nanoscale.

As a final set of examples, we extend our observations regarding the crucial importance of many-body vdW interactions (43) to the description of complex nanostructures such as carbon nanotubes and biological systems. As Fig. 6 demonstrates, the decay rate of the MBD interaction energy between a nanowire and the 1MC5 protein (human glutathione-dependent formaldehyde dehydrogenase) is much slower than the prediction of the pairwise-additive approximation. In addition, the ratio between the MBD and pairwise interaction energies, $\Delta E_{\mathrm{vdW}}^{\mathrm{LR}} / \Delta E_{\mathrm{vdW}}^{\mathrm{LR}(2)}$, is quite large and grows as a function of the wire-protein separation, demonstrating a nontrivial coupling between delocalized charge density fluctuations sustained across the protein and along the wire.

\section{Outlook}

Our analysis demonstrates the possibilities for engineering the $\mathrm{vdW}$ interaction energy power laws in low-dimensional nanostructures by modifying their underlying dimensionality, topology, and response properties. Moreover, our findings suggest that complex power laws could also play an important role in numerous low-dimensional biologically relevant systems, including phospholipid aggregates and bilayers (44) or even the subnanometer spatula-shaped structures that determine the peculiar pedal adhesion in the gecko (45). Our study provides strong evidence that the ubiquitous vdW forces between polarizable nonmetallic nanostructures can be more completely understood in terms of collective interactions between wavelike charge density fluctuations, rather than simply a summation over pairwise interactions between instantaneous particle- or fragment-like dipolar fluctuations.

In a manner analogous to metallic systems, the marked nonlocality of the valence electronic response in low-dimensional polarizable nanostructures is reflected in the emergence of collective modes that span the entire system. It is these delocalized wavelike charge density fluctuations that are responsible for the nontrivial interaction energy power laws observed herein that substantially deviate from the predictions of standard pairwise-additive vdW approximations. As such, these wavelike fluctuations govern the magnitude of vdW interactions at large, but finite, distances of relevance at the nanoscale. Our findings reveal a smooth transition from a system consisting of independent atoms to the collective fully interacting limit, thereby providing a potential pathway for tuning the fundamental noncovalent vdW interactions responsible for the assembly of complex polarizable nanostructures.

\section{REFERENCES AND NOTES}

1. V. A. Parsegian, Van der Waals Forces: A Handbook for Biologists, Chemists, Engineers, and Physicists (Cambridge Univ. Press, 2006)

2. J. F. Dobson, Int. J. Quantum Chem. 114, 1157-1161 (2014).

3. A. Tkatchenko, Adv. Funct. Mater. 25, 2054-2061 (2015).

4. A. M. Reilly, A. Tkatchenko, Chem. Sci. 6, 3289-3301 (2015). 
5. Y. V. Shtogun, L. M. Woods, . J. Phys. Chem. Lett. 1, 1356-1362 (2010).

6. W. Gao, A. Tkatchenko, Phys. Rev Lett 114, 096101 (2015)

7. A. M. Reilly, A. Tkatchenko, Phys. Rev. Lett. 113, 055701 (2014).

8. N. Ferri, R. A. DiStasio Jr., A. Ambrosetti, R. Car, A. Tkatchenko, Phys. Rev. Lett. 114, 176802 (2015).

9. A. J. Stone, The Theory of Intermolecular Forces (Oxford Univ. Press, ed. 2, 2013).

10. E. M. Lifshitz, Sov. Phys. JETP 2, 73 (1956).

11. S. Grimme, WIREs Comput. Mol. Sci. 1, 211-228 (2011)

12. A. Tkatchenko, M. Scheffler, Phys. Rev. Lett. 102, 073005 (2009).

13. C. A. Silvera Batista, R. G. Larson, N. A. Kotov, Science 350 , 1242477 (2015)

14. P. Loskill et al., Adv. Colloid Interface Sci. 179-182, 107-113 (2012).

15. P. Loskill et al., J. R. Soc. Interface 10, 20120587 (2013).

16. S. Tsoi et al., ACS Nano 8, 12410-12417 (2014).

17. G. A. Rance, D. H. Marsh, S. J. Bourne, T. J. Reade, A. N. Khlobystov, ACS Nano 4, 4920-4928 (2010).

18. C. Wagner et al., Nat. Commun. 5, 5568 (2014).

19. D. B. Chang, R. L. Cooper, J. E. Drummond, A. C. Young, Phys. Lett. A 37, 311-312 (1971).

20. M. Boström, B. E. Sernelius, Phys. Rev. B 61, 2204-2210 (2000)

21. J. F. Dobson, A. White, A. Rubio, Phys. Rev. Lett. 96, 073201 (2006).

22. J. F. Dobson, T. Gould, G. Vignale, Phys. Rev. X 4, 021040 (2014).

23. A. J. Misquitta, J. Spencer, A. J. Stone, A. Alavi, Phys. Rev. B 82, 075312 (2010).

24. A. J. Misquitta, R. Maezono, N. D. Drummond, A. J. Stone, R. J. Needs, Phys. Rev. B 89, 045140 (2014)

25. A. Tkatchenko, R. A. DiStasio Jr., R. Car, M. Scheffler, Phys. Rev. Lett. 108, 236402 (2012)

26. J. Applequist, K. R. Sundberg, M. L. Olson, L. C. Weiss, J. Chem. Phys. 70, 1240 (1979).

27. W. L. Bade, J. Chem. Phys. 27, 1280 (1957).

28. A. G. Donchev, J. Chem. Phys. 125, 074713 (2006).

29. A. Ambrosetti, A. M. Reilly, R. A. DiStasio Jr., A. Tkatchenko, J. Chem. Phys. 140, 18A508 (2014)

30. A. Tkatchenko, A. Ambrosetti, R. A. DiStasio Jr., J. Chem. Phys. 138, 074106 (2013)

31. A. P. Jones, J. Crain, V. P. Sokhan, T. W. Whitfield, G. J. Martyna, Phys. Rev. B 87, 144103 (2013).

32. A. M. Reilly, A. Tkatchenko, J. Phys. Chem. Lett. 4, 1028-1033 (2013).

33. A. Ambrosetti, D. Alfè, R. A. DiStasio Jr., A. Tkatchenko, J. Phys. Chem. Lett. 5, 849-855 (2014)

34. R. A. DiStasio Jr., O. A. von Lilienfeld, A. Tkatchenko, Proc. Natl. Acad. Sci. U.S.A. 109, 14791-14795 (2012)

35. R. A. DiStasio Jr., V. V. Gobre, A. Tkatchenko, J. Phys. Condens. Matter 26, 213202 (2014).

36. J. F. Dobson, J. Wang, B. P. Dinte, K. McLennan, H. M. Le, Int. J. Quantum Chem. 101, 579-598 (2005).

37. V. V. Gobre, A. Tkatchenko, Nat. Commun. 4, 2341 (2013).

38. R.-F. Liu, J. G. Ángyán, J. F. Dobson, J. Chem. Phys. 134 114106 (2011).

39. M. Dion, H. Rydberg, E. Schröder, D. C. Langreth, B. I. Lundqvist, Phys. Rev. Lett. 92, 246401 (2004).

40. K. Berland et al., Rep. Prog. Phys. 78, 066501 (2015).

41. J. Dai, J. Yuan, P. Giannozzi, Appl. Phys. Lett. 95, 232105 (2009)

42. P. A. Denis, Chem. Phys. Lett. 508, 95-101 (2011).

43. J. Tao, J. P. Perdew, J. Chem. Phys. 141, 141101 (2014).

44. L. J. Lis, M. McAlister, N. Fuller, R. P. Rand, V. A. Parsegian, Biophys. J. 37, 667-672 (1982).

45. K. Autumn et al., Nature 405, 681-685 (2000)

46. See supplementary materials on Science Online.

\section{ACKNOWLEDGMENTS}

Supported by a startup grant from Cornell University (R.A.D.), European Research Council Starting Grant VDW-CMAT, and DFG/ SFB-951 HIOS project A10. A.A. thanks F. Toigo and P. L. Silvestrelli for useful discussions. This research used resources of the Argonne Leadership Computing Facility at Argonne National Laboratory, which is supported by the Office of Science of the U.S. Department of Energy under contract DE-AC02-06CH11357.

\section{SUPPLEMENTARY MATERIALS}

www.sciencemag.org/content/351/6278/1171/suppl/DC1

Theoretical Methods

Supplementary Text

Tables S1 to S3

10 December 2015; accepted 31 January 2016

10.1126/science.aae0509

REPORTS

QUANTUM OPTICS

\title{
Generation of multiphoton entangled quantum states by means of integrated frequency combs
}

\author{
Christian Reimer, ${ }^{1 *}$ Michael Kues, ${ }^{1 *} \dagger$ Piotr Roztocki, ${ }^{1}$ Benjamin Wetzel,,${ }^{1,2}$ \\ Fabio Grazioso, ${ }^{1}$ Brent E. Little, ${ }^{3}$ Sai T. Chu, ${ }^{4}$ Tudor Johnston, ${ }^{1}$ Yaron Bromberg, ${ }^{5} \ddagger$ \\ Lucia Caspani, ${ }^{6} \S$ David J. Moss, ${ }^{7}||$ Roberto Morandotti ${ }^{1,8} \dagger$
}

Complex optical photon states with entanglement shared among several modes are critical to improving our fundamental understanding of quantum mechanics and have applications for quantum information processing, imaging, and microscopy. We demonstrate that optical integrated Kerr frequency combs can be used to generate several bi- and multiphoton entangled qubits, with direct applications for quantum communication and computation. Our method is compatible with contemporary fiber and quantum memory infrastructures and with chip-scale semiconductor technology, enabling compact, low-cost, and scalable implementations. The exploitation of integrated Kerr frequency combs, with their ability to generate multiple, customizable, and complex quantum states, can provide a scalable, practical, and compact platform for quantum technologies.

M

ulti-entangled states of light hold answers to fundamental questions in quantum physics and are the cornerstone of a range of applications, including quantum communications (1), computation (2-4), and sensing and imaging with a resolution beyond the classical limit (5). Thus, the controllable realization of multiple quantum states in a compact platform would enable a practical and powerful implementation of quantum technologies. Although applications of frequency combs have been mostly classical thus far, their distinctive architecture, based on multiple interacting modes and the phase characteristics of the underlying nonlinear processes, has the potential to offer new and powerful ways to achieve the gener-

${ }^{1}$ Institut National de la Recherche Scientifique-Énergie Matériaux Télécommunications, 1650 Boulevard LionelBoulet, Varennes, Québec J3X 1S2, Canada. ²Department of Physics and Astronomy, University of Sussex, Falmer, Brighton BN1 9RH, UK. ${ }^{3}$ State Key Laboratory of Transient Optics and Photonics, Xi'an Institute of Optics and Precision Mechanics, Chinese Academy of Science, Xi'an, China. ${ }^{4}$ Department of Physics and Materials Science, City University of Hong Kong, Tat Chee Avenue, Hong Kong, China. ${ }^{5}$ Department of Applied Physics, Yale University, New Haven, CT 06520, USA. ${ }^{6}$ School of Engineering and Physical Sciences, Heriot-Watt University, Edinburgh EH14 4AS, UK ${ }^{7}$ School of Electrical and Computer Engineering, RMIT University, Melbourne, Victoria 3001, Australia. ${ }^{8}$ Institute of Fundamental and Frontier Sciences, University of Electronic Science and Technology of China, Chengdu 610054, China. *These authors contributed equally to this work. †Corresponding author. E-mail: michael.kues@emt.inrs.ca (M.K.); morandotti@ emt.inrs.ca (R.M.) ‡Present address: Racah Institute of Physics, The Hebrew University of Jerusalem, Jerusalem 91904, Israel. §Present address: Institute of Photonics, Department of Physics, University of Strathclyde, Glasgow G4 ONW, UK. ||Present address: Center for Micro-Photonics, Swinburne University of Technology, Hawthorn, Victoria 3122,Australia. ation of multiple, customizable, and complex states of nonclassical light. The quantum properties of frequency combs have recently begun to be investigated, revealing their potential for the generation of large quantum states $(6-8)$. However, the continuous-variable nonclassical states (squeezed vacuum) that have been demonstrated with this approach have not yet achieved the quality (amount of squeezing) required for optical quantum computation (9). For the generation of single photons and continuous- and discrete-variable quantum states (qubits), a wide variety of second- and third-order nonlinear sources, optical fibers, and gases, as well as single quantum emitters, have been exploited $(10,11)$. Recent progress has focused on transferring both classical frequency combs (12) and quantum sources (13) to integrated optical platforms. Such integrated approaches provide the advantages of compact, scalable, mass-producible, and lowcost devices (14). Demonstrated integrated devices include sources of heralded single photons (15-17) and entangled photon pairs (18), in principle allowing implementations of quantum algorithms $(19,20)$. Here we show the parallel generation of bi- and multiphoton entangled states in a compact, integrated quantum frequency comb source.

Our quantum frequency comb is generated in a CMOS (complementary metal-oxide semiconductor)compatible, high-refractive-index glass in a fourport microring resonator architecture [details on device fabrication and characteristics are presented in (21)]. The weak and anomalous dispersion of our device enables broadband phase matching for spontaneous four-wave mixing (SFWM), thereby generating a broad frequency 
This copy is for your personal, non-commercial use only.

If you wish to distribute this article to others, you can order high-quality copies for your colleagues, clients, or customers by clicking here.

Permission to republish or repurpose articles or portions of articles can be obtained by following the guidelines here.

The following resources related to this article are available online at www.sciencemag.org (this information is current as of March 10, 2016 ):

Updated information and services, including high-resolution figures, can be found in the online version of this article at:

/content/351/6278/1171.full.html

Supporting Online Material can be found at:

/content/suppl/2016/03/09/351.6278.1171.DC1.html

This article cites $\mathbf{4 3}$ articles, 2 of which can be accessed free:

/content/351/6278/1171.full.html\#ref-list-1

This article appears in the following subject collections:

Chemistry

/cgi/collection/chemistry 